\title{
PROPRIEDADES FÍSICAS DO BAMBU-MOSSÔ (Phyllostachys pubescens Mazel ex H. de Lehaie) EM DIFERENTES IDADES E POSIÇÕES DO COLMO
}

\author{
Rodrigo Strzelecki Berndsen ${ }^{1}$, Ricardo Jorge Klitzke ${ }^{2}$, Djeison Cesar Batista ${ }^{3}$, \\ Eduardo Mauro do Nascimento ${ }^{4}$, Fabiano Ostapiv ${ }^{4}$ \\ ${ }^{1}$ Tecnólogo Mecânico, M.Sc., Depto. de Design de Produto, UP, Curitiba, PR, Brasil - rodrigoberndsen@ yahoo.com.br \\ ${ }^{2}$ Eng. Florestal, Dr., Depto. de Engenharia e Tecnologia Florestal, UFPR, Curitiba, PR, Brasil - rklitzke@ufpr.br \\ ${ }^{3}$ Eng. Florestal, M.Sc., Doutorando em Engenharia Florestais, UFPR, Curitiba, PR, Brasil - djeison1984@ gmail.com \\ ${ }^{4}$ Eng. Mecânico, Dr., Depto. de Engenharia Mecânica UTFPR, Curitiba, PR, Brasil - nascimento@utfpr.edu.br, ostapiv@utfpr.edu.br
}

Recebido para publicação: 10/07/2008 - Aceito para publicação: 29/04/2009

\begin{abstract}
Resumo
As buscas por materiais renováveis de baixo impacto ambiental apontam o bambu como um material que promove o desenvolvimento sustentável devido ao seu rápido crescimento e por não precisar ser replantado após seu corte. O principal objetivo deste estudo foi determinar as seguintes propriedades físicas do bambu-mossô (Phyllostachys pubescens Mazel ex H. de Lehaie): teor de umidade, densidade básica e retratibilidade. Procurou-se investigar as diferenças entre as propriedades físicas em diferentes posições de colmos (base, meio, topo) de diferentes idades (1, 3 e 5 anos), visando a melhor utilização dessa matéria-prima. Os resultados mostraram que não houve interação significativa entre os fatores "idade" e "posição" para as propriedades teor de umidade e densidade básica, de acordo com a ANOVA em arranjo fatorial (95\% de probabilidade). $\mathrm{O}$ teor de umidade diminuiu com o aumento da idade e da base para o topo, enquanto que a densidade básica aumentou com a idade e da base para o topo. A interação significativa entre os fatores "idade" e "posição" somente foi observada para a retratibilidade. A porção menos estável foi o topo dos bambus com cinco anos de idade, onde se encontraram maiores valores para a contração radial, tangencial e volumétrica. A porção basal na idade de três anos revelou-se a mais estável em contração volumétrica e tangencial. As porções base e topo na idade de um ano foram as mais estáveis quanto à contração radial.

Palavras-chave: Phyllostachys pubescens; bambu-mossô; propriedades físicas.
\end{abstract}

\begin{abstract}
Physical properties of moso bamboo (Phyllostachys pubescens Mazel ex H. de Lehaie) at different ages and culm positions. The searching for renewable material with low environment impact put bamboo forward as a material that promotes the sustainable development due to its fast growing and that is not necessary to replant after harvesting. The aim of this study was to evaluate the following physical properties at different culm positions (base, middle and top) and ages (1, 3 and 5 years old) of moso bamboo (Phyllostachys pubescen Mazel ex H. de Lehaie): moisture content, basic density and shrinkage. The results showed that there was no significant interaction between the factors "age" and "position" for moisture content and basic density according to ANOVA in factorial arrange (95\% of probability). Moisture content decreased with the increase of age and from base to top, while basic density increased with age and from base to top. Significant interaction between the factors "age" and "position" was only observed for shrinkage test according to ANOVA in factorial arrange. The portion less stable was the top of bamboo with five years old, which presented the highest radial, tangential and volumetric shrinkages. The basal portion of three years old was the most stable in volumetric and tangential shrinkage. The portions of base and top of one year old were the most stable in radial shrinkage.

Keywords: Phyllostachys pubescens; moso bamboo; physical properties.
\end{abstract}

\section{INTRODUÇÃO}

Nos últimos anos, o bambu tem sido redescoberto como um importante material renovável, com grande potencial de utilização pelas indústrias de transformação secundária, agregando valor à matériaprima. As indústrias de produtos de bambu resultarão num alívio da pressão sobre as florestas, como 
também criarão empregos e oportunidades para pessoas do campo. A área plantada com bambu na China é de cerca de 20 milhões de hectares. Com exceção da Europa, os bambus ocorrem naturalmente nas regiões tropicais, subtropicais e temperadas de todos os países (CHUNG, 2003a).

Segundo Kleinhenz; Midmore (2001), na Ásia, a taxa de consumo per capita de bambu é de 12 $\mathrm{kg}$ de produtos por ano, e estima-se o consumo de 20 milhões de ton/ano de produtos de bambu na China. Beraldo et al. (1998) afirmam que, só na China, o potencial de produção de compensados de bambu é de $4.200 .000 \mathrm{~m}^{3}$, o que corresponde a quatro vezes sua produção atual de madeira. Esse potencial decorre da grande área replantada com bambu naquele país.

Segundo Beraldo; Azzini (2004), no Brasil, o bambu, apesar de suas amplas possibilidades de utilização como material fibroso, é pouco utilizado em comparação às espécies arbóreas ou madeireiras, que representam a quase totalidade da matéria-prima fibrosa empregada nas mais variadas utilizações, desde a queima para geração de energia de uso doméstico até a produção industrial de celulose e papel. A crescente escassez e a valorização da madeira observada nos últimos anos têm contribuído para aumentar o grande interesse do bambu como matéria-prima.

O bambu Phyllostachys pubescens, vulgarmente conhecido em todo o mundo como "mossô", é a espécie mais importante da China, onde cobre uma área de 3 milhões de hectares, ou cerca de $2 \%$ do total da sua área florestal. É a principal espécie para a produção de bambu laminado colado e broto de bambu, e tem um importante papel para o meio ambiente naquele país. Pode crescer mais de $119 \mathrm{~cm}$ em 24 horas e 24 metros de altura em 40 a 50 dias (CHUNG, 2003a).

Devido a fatores climáticos, a espécie mais recomendada como alternativa de reflorestamento no sul do Brasil é também a Phyllostachys pubescens. Por isso faz-se necessário compreender suas propriedades, a fim de se viabilizar a utilização dessa espécie como uma alternativa para suprir o déficit da produção de madeira, contribuindo para promover o desenvolvimento sustentável.

O principal objetivo deste estudo foi determinar as propriedades físicas - teor de umidade verde, densidade básica e retratibilidade - do bambu-mossô (Phyllostachys pubescens Mazel ex H. de Lehaie). Foram investigadas as diferenças entre os valores das propriedades físicas em diferentes posições do colmo (base, meio e topo) e diferentes idades (1, 3 e 5 anos), visando a melhor utilização dessa matériaprima.

\section{MATERIAL E MÉTODOS}

Os colmos de bambu-mossô utilizados neste estudo foram coletados na colônia Parque Verde, pertencente ao município de Fazenda Rio Grande, estado do Paraná, localizada a $25^{\circ} 40^{\prime}$ S e $49^{\circ} 19,50^{\prime}$ W. O diâmetro basal médio dos colmos escolhidos foi de $12 \mathrm{~cm}$ (retirado a aproximadamente 1,3 $\mathrm{m}$ de altura do solo) e a média das alturas foi de 17 metros. Foram coletados quatro colmos de cada idade $(1,3$ e 5 anos), totalizando 12 colmos. No entanto, depois de colhidos, utilizaram-se apenas os cinco primeiros metros dos colmos. Dessas seções de 5 metros, utilizaram-se apenas as partes denominadas "base", "meio" e "topo", de acordo com a figura 1.

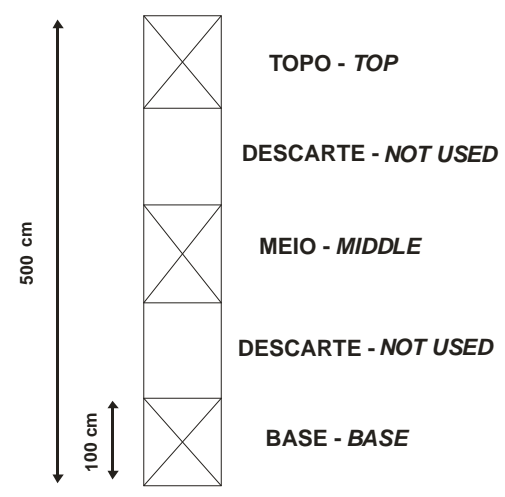

Figura 1. Partes do colmo estudadas - base, meio e topo.

Figure 1. Studied parts of the culm - base, middle e top. 
Depois de definidas conforme a figura 1, as partes dos colmos foram cortadas longitudinalmente em três secções iguais de $120^{\circ}$ (Figura 2) em serra de fita simples no Laboratório de Usinagem da Madeira da Universidade Federal do Paraná. De cada seção foi extraída uma ripa de $30 \mathrm{~mm}$ de largura, que foi utilizada para a confecção dos corpos-de-prova. Posteriormente, foram retiradas as saliências dos nós das faces interna e externa, com o auxílio de uma lixadeira. As ripas foram passadas em uma máquina sepilhadora, aplainando-se as duas faces. Imediatamente após essa pequena usinagem, os ensaios foram realizados.

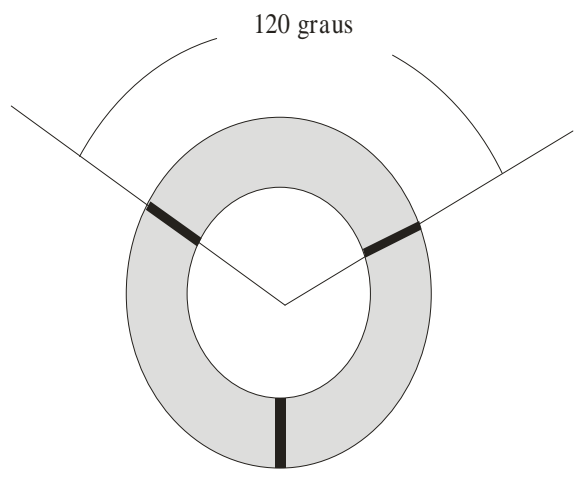

Figura 2. Esquema da divisão da seção transversal dos colmos.

Figure 2. Culms' transversal section division scheme.

\section{Teor de umidade}

O teor de umidade foi obtido de acordo com a norma ASTM D 143-06, exceto pelas dimensões dos corpos-de-prova. Os cálculos foram feitos de acordo com a equação 1.

$$
\mathrm{U}=[(\mathrm{MU}-\mathrm{MS}) / \mathrm{MS}] \times 100
$$

Em que: U: teor de umidade (\%);

MU: massa úmida $(\mathrm{g})$;

MS: massa seca $(\mathrm{g})$.

Os corpos-de-prova apresentavam as dimensões de 20 x $40 \mathrm{~mm}$, com a espessura variável, de acordo com o colmo.

\section{Densidade básica}

A densidade básica foi obtida de acordo com a norma ASTM D 143-06, exceto pelas dimensões dos corpos-de-prova. Os cálculos foram feitos de acordo com a equação 2.

$$
\rho b=M S / V V
$$

Em que: $\rho$ b: densidade básica $\left(\mathrm{g} / \mathrm{cm}^{3}\right)$;

MS: massa seca $(\mathrm{g})$;

VV: volume verde $\left(\mathrm{cm}^{3}\right)$.

Os corpos de prova tinham as dimensões de 20 x $40 \mathrm{~mm}$, com a espessura variável, de acordo com o colmo.

\section{Ensaio de retratibilidade}

A estabilidade dimensional foi obtida de acordo com o ensaio de retratibilidade, que foi realizado de acordo com a norma COPANT 30: 1-005 (1972), adaptando-se as dimensões dos corpos de prova, 
padronizadas em $5 \times 5 \times 20 \mathrm{~mm}$. Para padronizar a espessura, o material foi removido a partir da face interna do bambu, para se obter em todos os corpos de prova a espessura desejada.

No ensaio de retratibilidade, foi determinada a contração máxima (desde a condição verde até anidra) das amostras. Como o colmo do bambu não é orientado por anéis de crescimento e raios (como a madeira), a determinação das contrações longitudinais radial e tangencial foi feita de acordo com o esquema proposto por Ahmad (2000), exemplificado na figura 3, em que a espessura do corpo-de-prova corresponde à direção estrutural radial e a largura corresponde à direção tangencial.

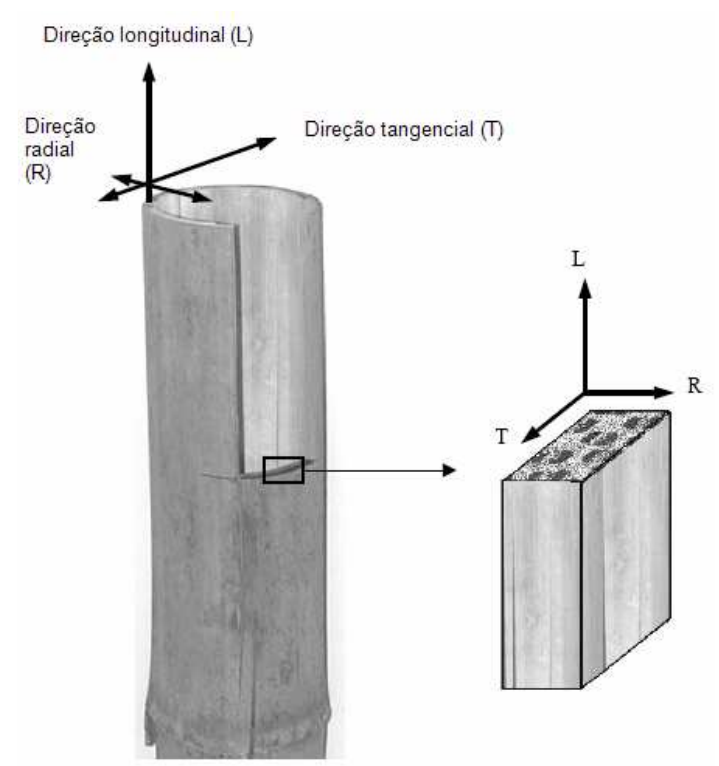

Figura 3. Direções estruturais do tecido do colmo do bambu.

Figure 3. Structural directions of bamboo tissue.

A contração máxima foi calculada de acordo com a equação 3.

$$
\left.\mathrm{C}=\left[\left(\mathrm{D}_{\mathrm{v}}-\mathrm{D}_{\mathrm{s}}\right) / \mathrm{D}_{\mathrm{v}}\right)\right] \times 100
$$

E que: C: contração máxima (\%);

$\mathrm{D}_{\mathrm{v}}$ : dimensão verde $(\mathrm{mm})$;

$\mathrm{D}_{\mathrm{s}}$ : dimensão seca, condição anidra (mm).

Foi utilizada uma estufa de laboratório sem ventilação forçada e uma balança de precisão com centésimos de grama para obtenção do peso seco dos corpos de prova.

\section{Análise estatística}

Na tabela 1, encontram-se definidos os tratamentos resultantes das combinações entre as idades e as posições estudadas.

Para cada tratamento, foram ensaiados 18 corpos de prova.

A análise de variância (ANOVA) em ensaio fatorial (95\% de probabilidade) foi aplicada às médias dos tratamentos, para se observar se houve diferença estatisticamente significativa entre eles. Foram combinados dois fatores em três níveis, idade (A) e posição (B), resultando em um fatorial $3 \times 3 \mathrm{e}$ a possível interação entre ambos. Quando a hipótese da nulidade foi rejeitada, aplicou-se o teste de Tukey para a diferenciação das médias. 
Tabela 1. Tratamentos.

Table 1. Treatments.

\begin{tabular}{lcc}
\hline Tratamento & Idade (anos) & Posição \\
\hline 1 & 1 & BASE \\
2 & 1 & MEIO \\
3 & 1 & TOPO \\
4 & 3 & BASE \\
5 & 3 & MEIO \\
6 & 3 & TOPO \\
7 & 5 & BASE \\
8 & 5 & MEIO \\
9 & 5 & TOPO \\
\hline
\end{tabular}

\section{RESULTADOS E DISCUSSÃO}

\section{Teor de umidade}

Os resultados obtidos pela ANOVA (95\% de probabilidade) demonstraram não haver interação significativa entre os fatores "idade" e "posição" $\left(\mathrm{F}=0,92^{\mathrm{ns}}\right)$ para o teor de umidade. Dessa maneira, analisaram-se os fatores separadamente. Os valores médios obtidos para o teor de umidade do bambumossô nas três idades e nas três posições encontram-se nas tabelas 2 e 3, respectivamente.

Tabela 2. Diferença entre as médias do teor de umidade por idade.

Table 2. Difference among the averages of moisture content per age.

\begin{tabular}{lccc}
\hline Idade & Teor de umidade (\%) & Desvio padrão (\%) & Coeficiente de variação $(\boldsymbol{\%})$ \\
\hline 1 & $90,71 \mathrm{a}$ & 15,89 & 17,52 \\
3 & $75,15 \mathrm{~b}$ & 14,63 & 19,46 \\
5 & $60,40 \mathrm{c}$ & 12,54 & 20,75 \\
\hline \multicolumn{2}{l}{ Médias seguidas por uma mesma letra em uma mesma coluna não diferem entre si de acordo com o teste de Tukey a $95 \%$ de } \\
probabilidade.
\end{tabular}

Tabela 3. Diferença entre as médias do teor de umidade por posição.

Table 3. Difference among the averages of moisture content per position.

\begin{tabular}{lccc}
\hline Posição & Teor de umidade $(\boldsymbol{\%})$ & Desvio padrão $(\boldsymbol{\%})$ & Coeficiente de variação $(\boldsymbol{\%})$ \\
\hline Base & $85,82 \mathrm{a}$ & 20,19 & 23,52 \\
Meio & $72,62 \mathrm{ab}$ & 16,95 & 23,34 \\
Topo & $67,82 \mathrm{~b}$ & 15,20 & 22,41 \\
\hline Médias seguidas por uma mesma letra em uma mesma coluna não diferem entre si de acordo com o teste de Tukey a 95\% de \\
probabilidade.
\end{tabular}

Análise da umidade por idade

A ANOVA aplicada entre as diferentes idades mostrou a existência de diferença significativa $\left(\mathrm{F}=25,41^{* *}\right)$ entre as médias do teor de umidade, quando agrupados por idade.

Os elevados coeficientes de variação obtidos se devem provavelmente ao número de colmos estudados. Com o aumento do número de colmos, o coeficiente tende a reduzir. Pode ser observado que ocorreu uma redução da umidade com a idade do bambu.

Observa-se que há uma correlação negativa entre o teor de umidade e a idade do bambu-mossô. O menor valor médio obtido foi de $60,40 \%$ nos colmos de cinco anos, enquanto que o maior foi de $90,71 \%$, obtido nos colmos de um ano. Em relação à idade, os teores de umidade médios deste trabalho foram inferiores àqueles observados por Chung (2003b), que obteve 135\% para um ano de idade, $91 \%$ para três e $82 \%$ para cinco anos.

Segundo Chung (2003b), a diminuição do teor de umidade médio devido ao aumento da idade deve-se provavelmente à diminuição do diâmetro do lúmen, provocado pelo aumento da espessura da parede das fibras, com ocorrência de maior concentração de extrativos no interior dos vasos, fazendo com que haja menos espaço para a água se alojar. 
Análise da umidade por posição

A ANOVA aplicada entre as diferentes posições mostrou a existência de diferença significativa $(\mathrm{F}=9,62 * *)$ entre as médias do teor de umidade quando agrupados por posição no colmo.

Foram obtidos elevados coeficientes de variação para o teor de umidade em relação à posição dos colmos, semelhante ao resultado obtido por idade, devido às mesmas razões anteriormente citadas, com tendência de redução da umidade inversamente proporcional à altura do bambu.

A base dos colmos de bambu-mossô apresentou teor de umidade médio superior ao do topo (85,82\% contra $67,82 \%)$, no entanto a porção média dos colmos não diferiu estatisticamente nem da base nem do topo, assumindo um valor intermediário entre eles $(72,62 \%)$.

Quanto à posição, estes resultados foram inferiores àqueles obtidos por $\mathrm{Li}$ (2004), que observou teores de umidade médios de $82 \%$ na parte superior e $110 \%$ na parte basal dos colmos.

Foi observado que a densidade básica média aumenta com a posição do colmo, explicando a redução da umidade da base para o topo com a diminuição dos lumes e aumento da espessura das paredes dos feixes fibrovasculares.

O teor de umidade também é influenciado pelo solo, condições climáticas e precipitação pluviométrica, entre outros fatores, mostrando assim variações conforme o local da colheita.

\section{Densidade básica}

Análise geral

De acordo com a ANOVA em arranjo fatorial, a interação entre os fatores "idade" e "posição" não foi significativa ao nível de $95 \%$ de probabilidade $\left(\mathrm{F}=0,16^{\mathrm{ns}}\right)$. Optou-se, então, por analisarem-se os fatores separadamente.

Análise da densidade por idade

A ANOVA demonstrou haver diferença significativa entre as médias para os valores da densidade básica das idades $\left(\mathrm{F}=31,55^{* *}\right)$. Na tabela 4 , encontra-se o resultado do teste de diferenciação de médias por idade.

Tabela 4. Diferença entre as médias de densidade básica por idade.

Table 4. Difference among the averages of basic density per age.

\begin{tabular}{|c|c|c|c|}
\hline Idade & Densidade básica média $\left(\mathrm{g} / \mathrm{cm}^{3}\right)$ & Desvio padrão $\left(\mathrm{g} / \mathrm{cm}^{3}\right)$ & Coeficiente de variação $(\%)$ \\
\hline 1 & $0,60 \mathrm{a}$ & 0,06 & 10,16 \\
\hline 3 & $0,67 \mathrm{~b}$ & 0,07 & 10,01 \\
\hline 5 & $0,76 \mathrm{c}$ & 0,07 & 9,03 \\
\hline
\end{tabular}

De acordo com a tabela 4 , as médias de densidade básica dos colmos de um, três e cinco anos foram, respectivamente, $0,60 \mathrm{~g} / \mathrm{cm}^{3}, 0,67 \mathrm{~g} / \mathrm{cm}^{3}$ e $0,76 \mathrm{~g} / \mathrm{cm}^{3}$. Pode-se distinguir claramente a diferença entre as médias e a correlação positiva entre idade e densidade básica dos colmos do bambu-mossô.

Esses valores foram superiores àqueles obtidos por Chung (2003b), que, para colmos de um, três e cinco anos, obteve valores respectivos de $0,42 \mathrm{~g} / \mathrm{cm}^{3}, 0,61 \mathrm{~g} / \mathrm{cm}^{3}$ e $0,62 \mathrm{~g} / \mathrm{cm}^{3}$. Essa diferença entre resultados está correlacionada, provavelmente, com as variações edafoclimáticas das regiões de estudo.

O aumento da densidade básica média com a maturação do bambu ocorre, provavelmente, pelo aumento na espessura da parede das células dos feixes fibrovasculares e pela consequente diminuição do lúmen.

Análise por posição

A ANOVA demonstrou haver diferença significativa entre as médias de densidade básica nas diferentes posições $\left(\mathrm{F}=8,38^{* *}\right)$. Na tabela 5 , encontra-se o resultado do teste de diferenciação de médias por posição.

A densidade básica média aumentou em direção ao topo dos colmos de bambu-mossô. No entanto não houve diferença estatística entre a densidade média do topo e do meio dos colmos. 
Segundo Chung (2003b), o aumento da densidade básica média na direção base-topo ao longo dos colmos é justificado pelo aumento da concentração dos feixes fibrovasculares, dado pela diminuição do tecido parenquimático.

Tabela 5. Diferença entre as médias de densidade básica por posição.

Table 5. Difference among averages of basic density per position.

\begin{tabular}{lccc}
\hline Posição & Densidade básica média $\left(\mathbf{g} / \mathbf{c m}^{3}\right)$ & Desvio padrão & Coeficiente de variação $(\%)$ \\
\hline Base & $0,63 \mathrm{a}$ & 0,09 & 13,74 \\
Meio & $0,69 \mathrm{~b}$ & 0,09 & 12,75 \\
Topo & $0,71 \mathrm{~b}$ & 0,09 & 12,02 \\
\hline
\end{tabular}

Médias seguidas por uma mesma letra em uma mesma coluna não diferem entre si de acordo com o teste de Tukey a 95\% de probabilidade.

\section{Ensaio de retratibilidade}

$\mathrm{Na}$ tabela 6, encontram-se os resultados do teste "F" gerados pela ANOVA ao analisar-se a interação entre os fatores "idade" e "posição" no ensaio de retratibilidade. Os resultados demonstraram interação significativa entre os fatores para esse ensaio.

Tabela 6. Valores de "F" para as interações entre os fatores "idade" e "posição" no ensaio de retratibilidade.

Table 6. "F" values: interation between "age" and "position"in shrinkage test.

\begin{tabular}{lc}
\hline \multicolumn{2}{c}{ Interação idade e posição } \\
\hline Contração & Valor de f \\
\hline Radial & $3,77^{* *}$ \\
Tangencial & $7,29 * *$ \\
Volumétrica & $6,01 * *$ \\
\hline
\end{tabular}

Contração radial máxima

Na tabela 7, encontram-se os resultados do teste de Tukey aplicado às médias de contração radial máxima.

Tabela 7. Diferença entre as médias de contração radial máxima.

Table 7. Difference among averages of maximum radial shrinkage.

\begin{tabular}{lccc}
\hline \multirow{2}{*}{ Idade } & & Posição & Topo \\
\cline { 2 - 4 } & Base & Meio & 4,43 a A \\
\multirow{2}{*}{1} & $4,59 \mathrm{~b} \mathrm{~A}$ & $3,77 \mathrm{a} \mathrm{A}$ & $(4,33 \%)$ \\
& $(5,72 \%)$ & $(4,91 \%)$ & $4,83 \mathrm{a} \mathrm{A}$ \\
3 & $5,35 \mathrm{ab} \mathrm{A}$ & $5,23 \mathrm{~b} \mathrm{~A}$ & $(4,09 \%)$ \\
& $(6,97 \%)$ & $(5,03 \%)$ & $7,23 \mathrm{~b} \mathrm{~A}$ \\
& $6,07 \mathrm{a} \mathrm{B}$ & $6,43 \mathrm{c} \mathrm{AB}$ & $(3,63 \%)$ \\
\hline
\end{tabular}

Médias seguidas por uma mesma letra minúscula em uma mesma coluna não diferem entre si de acordo com o teste de Tukey a $95 \%$ de probabilidade. Médias seguidas por uma mesma letra maiúscula em uma mesma linha não diferem entre si de acordo com o teste de Tukey a 95\% de probabilidade (Coeficiente de variação).

O tratamento 9 (cinco anos de idade, topo) apresentou a maior média de contração radial máxima, com 7,23\%. No entanto, esse tratamento não diferiu estatisticamente do tratamento 8 (cinco anos, meio), com 6,43\%. Ainda para essa propriedade, o tratamento mais estável foi o 2 (um ano, meio), com $3,77 \%$, não diferindo, porém, do tratamento 3 (um ano, topo), com 4,43\%.

Chung (2003b) observou uma contração radial média de 8,7\% para a mesma espécie deste estudo, de 9,8\% para a espécie Dendrocalamus giganteus e de 5,0\% para a espécie Bambusa vulgaris. Garbino et al. (2004) obtiveram valores de $7,11 \%$ a $11,44 \%$ para a espécie Dendrocalamus giganteus. Adb Latif (1993) relata médias entre 5,4\% e 9,5\% para a espécie Bambusa bluemeana.

Mesmo o tratamento 9, que indicou a maior média absoluta para a contração radial máxima $(7,23 \%)$ foi inferior ao resultado obtido por Chung (2003b). A média geral da contração radial máxima 
foi de 5,27\%, equiparando-se às espécies Bambusa vulgaris. (CHUNG, 2003b) e Bambusa bluemeana (ADB LATIF, 1993), que são reconhecidamente mais estáveis que a espécie Phyllostachys pubescens.

Contração tangencial máxima

Na tabela 8 encontram-se os resultados do teste de diferenciação de médias aplicado aos resultados de contração tangencial máxima.

A maior média de contração tangencial máxima foi observada no tratamento 9 (cinco anos de idade, topo), com 7,52\%.

Tabela 8. Diferença entre as médias de contração tangencial máxima.

Table 8. Difference among averages of maximum tangential shrinkage.

\begin{tabular}{lccc}
\hline \multirow{2}{*}{ Idade } & \multicolumn{2}{c}{ Posição } \\
\cline { 2 - 4 } & Base & Meio & Topo \\
\hline \multirow{2}{*}{1} & $6,15 \mathrm{a} \mathrm{A}$ & $5,75 \mathrm{a} \mathrm{A}$ & $6,28 \mathrm{~b} \mathrm{~A}$ \\
& $(4,19 \%)$ & $(4,48 \%)$ & $(4,10 \%)$ \\
3 & $5,60 \mathrm{ab} \mathrm{A}$ & $6,24 \mathrm{a} \mathrm{A}$ & $6,36 \mathrm{~b} \mathrm{~A}$ \\
& $(4,60 \%)$ & $(4,13 \%)$ & $(4,05 \%)$ \\
5 & $5,09 \mathrm{~b} \mathrm{~B}$ & $5,31 \mathrm{a} \mathrm{B}$ & $7,52 \mathrm{a} \mathrm{A}$ \\
& $(5,06 \%)$ & $(4,85 \%)$ & $(3,43 \%)$ \\
\hline
\end{tabular}

Médias seguidas por uma mesma letra minúscula em uma mesma coluna não diferem entre si de acordo com o teste de Tukey a 95\% de probabilidade. Médias seguidas por uma mesma letra maiúscula em uma mesma linha não diferem entre si de acordo com o teste de Tukey a 95\% de probabilidade. (Coeficiente de variação).

O tratamento 7 obteve a menor média (cinco anos de idade, base), com 5,09\%, não diferindo estatisticamente do tratamento 4 (três anos de idade, base), com média igual a 5,60\%.

Chung (2003b) obteve contração tangencial máxima de 5,5\% para a mesma espécie deste trabalho; de 7,8\% para a espécie Dendrocalamus giganteus e de 4,7\% para a espécie Bambusa vulgaris. Garbino et al. (2004) obtiveram valores de $6,11 \%$ a $11,78 \%$ para a espécie Dendrocalamus giganteus. Adb Latif (1993) obteve médias de 6,4\% a 20,1\% para a espécie Bambusa bluemeana.

A média geral da contração tangencial máxima foi de $6,03 \%$, portanto próxima àquela relatada por Chung (2003b). No entanto, os tratamentos 7 e 8 apresentaram médias inferiores àquelas obtidas por esse autor.

Contração volumétrica máxima

Na tabela 9, encontram-se os resultados do teste de Tukey aplicado para a diferenciação das médias de contração volumétrica máxima.

Tabela 9. Diferença entre as médias de contração volumétrica máxima.

Table 9. Difference among averages of maximum volumetric shrinkage.

\begin{tabular}{lccc}
\hline \multirow{2}{*}{ Idade } & & Posição & Topo \\
\cline { 2 - 4 } & Base & Meio & $11,98 \mathrm{~b} \mathrm{~A}$ \\
1 & $10,44 \mathrm{a} \mathrm{B}$ & $10,75 \mathrm{ab} \mathrm{B}$ & $(3,44 \%)$ \\
& $(3,95 \%)$ & $(3,84 \%)$ & $12,54 \mathrm{~b} \mathrm{~A}$ \\
3 & $9,13 \mathrm{~b} \mathrm{C}$ & $11,27 \mathrm{a} \mathrm{B}$ & $(3,29 \%)$ \\
& $(4,52)$ & $(3,66 \%)$ & $14,06 \mathrm{a} \mathrm{A}$ \\
5 & $9,32 \mathrm{~b} \mathrm{~B}$ & $10,00 \mathrm{~b} \mathrm{~B}$ & $(2,93 \%)$ \\
\hline
\end{tabular}

Médias seguidas por uma mesma letra minúscula em uma mesma coluna não diferem entre si de acordo com o teste de Tukey a 95\% de probabilidade. Médias seguidas por uma mesma letra maiúscula em uma mesma linha não diferem entre si de acordo com o teste de Tukey a 95\% de probabilidade (Coeficiente de variação).

O tratamento 9 (cinco anos de idade, topo) obteve a maior média de contração volumétrica máxima, com $14,06 \%$. O tratamento 4 (três anos de idade, base) foi o mais estável, com a média de $9,13 \%$, não diferindo, no entanto, do tratamento 7 (cinco anos de idade, topo), com média de 9,32\%.

Chung (2003b) obteve média de contração máxima de 14,7\% para a mesma espécie deste estudo, de $19,7 \%$ para a espécie Dendrocalamus giganteus e de 10,2\% para a espécie Bambusa vulgaris. 
Portanto, mesmo o tratamento 9, que obteve a maior média absoluta deste estudo (14,06\%), foi mais estável que a média obtida por Chung (2003b) para a espécie Phyllostachys pubescens.

A média geral da contração volumétrica máxima dos tratamentos foi de $11,05 \%$, inferior àquela obtida por Chung (2003b) para a espécie Bambusa vulgaris (10,2\%), que é uma das espécies reconhecidamente mais estáveis dimensionalmente.

\section{CONCLUSÕES}

- Não houve interação significativa entre os fatores idade e posição para as propriedades físicas, de teor de umidade e densidade básica.

- O teor de umidade diminui com o aumento da idade, e da base para o topo.

- A densidade básica aumenta com a idade, e da base para o topo.

- Houve interação significativa entre os fatores idade e posição para retratibilidade.

- O tratamento menos estável foi o 9, correspondente ao topo dos colmos com cinco anos de idade, que obteve as maiores contrações máximas.

- A porção basal na idade de três anos (tratamento 4) obteve as menores médias de contração volumétrica e tangencial máximas. As porções base e topo, da idade de um ano (tratamentos 1 e 3), foram as mais estáveis quanto à contração radial máxima.

\section{REFERÊNCIAS}

ADB LATIF, M. Effects of age and height of three bamboo species on their machining properties. Journal of Tropical Forest Science, Kuala Lumpur, v. 5, n.4, p. 528-535, 1993.

AHMAD, M. Analysis of calcutta bamboo for structural composite materials. Dissertation, Wood Sci. and Forest Products, VT. pp. 210. 2000.

ASTM - AMERICAN SOCIETY FOR TESTING AND MATERIALS. D-143-94: Standard Test Methods for Small Clear Specimens of Timber. Conshohocken, PA, 2004. (Wood Annual Book of ASTM Standards, v. 04.10).

BERALDO, L.; AZZINI, A.; ZULLO, J.; Características físicas, químicas e anatômicas de três espécies de bambu gigantes. ENCONTRO BRASILEIRO EM MADEIRAS E ESTRUTURAS DE MADEIRA. 6., 1998, Florianópolis. Anais... Florianópolis: IBRAMEM, 1998. v. 4, p. 297-306.

BERALDO, A. L.; AZZINI, A. Bambu características e aplicações. Rio Grande do Sul: Guaíba: Agropecuária, 2004.

CHUNG, Z. F. The production and utilization of bamboo forest in China. Hangzhou: China National Research Center of Bamboo, 2003a.

Studies on Growth of bamboo shoots and culms. Hangzhou: China National Research Center of Bamboo, 2003b.

COMISSÃO PANAMERICANA DE NORMAS TÉCNICAS (COPANT). COPANT 462: Método de determinación de la contracción. Caracas, 1972.

GARBINO, L. V.; GONÇALVES, M. T. T.; PEREIRA M. A. R. Procedimentos experimentais para determinação de propriedades físicas do bambu. In: ENCONTRO BRASILEIRO EM MADEIRAS E EM ESTRUTURAS DE MADEIRA, 9., 2004, Cuiabá. Anais... Cuiabá: Universidade Federal do Mato Grosso, 2004.

KLEINHENZ, V.; MIDMORE, D. J. Aspects of bamboo agronomy. Austrália: Academic: Central Quensland University, 2001.

LI, X. Physical, chemical, and mechanical properties of bamboo and its utilization potential for fiberboard manufacturing. Dissertation, The School of Renewable Natural Resources, Louisiana, 2004. $76 \mathrm{p}$. 
